\title{
Échanges du phosphore à l'interface eau-sédiment dans un bassin de maturation (lagunage de Boujaad) et un étang d'alevinage (station de pisciculture Deroua, Béni-Mellal), Maroc \\ Phosphate exchanges at the water-sediment interface in a maturation pond (lagunage of Boujaad) and a fry pond (Deroua fisheries farm, Beni-Mellal), Morocco
}

\author{
M. Kdadri, M. Hasnaoui et L. Ait Brahim
}

Volume 18, numéro hors-série, 2005

URI : https://id.erudit.org/iderudit/705574ar

DOI : https://doi.org/10.7202/705574ar

Aller au sommaire du numéro

Éditeur(s)

Université du Québec - INRS-Eau, Terre et Environnement (INRS-ETE)

ISSN

0992-7158 (imprimé)

1718-8598 (numérique)

Découvrir la revue

Citer cet article

Kdadri, M., Hasnaoui, M. \& Ait Brahim, L. (2005). Échanges du phosphore à l'interface eau-sédiment dans un bassin de maturation (lagunage de Boujaad) et un étang d'alevinage (station de pisciculture Deroua, Béni-Mellal), Maroc. Revue des sciences de l'eau / Journal of Water Science, 18, 27-36.

https://doi.org/10.7202/705574ar
Résumé de l'article

Les sédiments utilisés dans cette étude sont prélevés dans deux milieux différents (bassin de maturation du lagunage de Boujaad et étang d'alevinage de la station Deroua, Béni-Mellal) dans le but de comprendre les conditions du milieu qui provoquent les échanges du phosphore à l'interface eau-sédiment et de comparer les échanges du phosphore à ce niveau.

Cette étude a montré une différence dans la composition chimique des deux sédiments. En effet, au niveau de l'étang d'alevinage, le phosphore inorganique représente une part majoritaire (92,5\%) surtout liée au calcium. En revanche, le phosphore total est réparti en phosphore organique $(49,2 \%)$ et inorganique $(49,8 \%)$ au niveau du bassin de maturation.

Dans le bassin de maturation, le relargage du phosphore est plus important et il est sous forme inorganique lié essentiellement aux hydroxydes de fer. La fixation du phosphore par les sédiments est importante particulièrement en conditions aérobies, aussi bien dans le bassin de maturation que dans l'étang d'alevinage. 


\title{
Échanges du phosphore à l'interface eau-sédiment dans un bassin de maturation (lagunage de Boujaad) et un étang d'alevinage (station de pisciculture Deroua, Béni-Mellal), Maroc
}

\author{
Phosphate exchanges at the water-sediment \\ interface in a maturation pond (lagunage of Boujaad) \\ and a fry pond (Deroua fisheries farm, Beni-Mellal), \\ Morocco
}

M. KDADRI ${ }^{1}$, M. HASNAOUI $^{1 \star}$, L. AIT BRAHIM ${ }^{2}$

Reçu le 10 novembre 2003, accepté le 7 janvier 2005**.

SUMMARY

Sediment samples used in this work were taken from two different sites (maturation pond of Boujaad lagunage and fry pond of the Deroua fish farm) in order to understand and compare the conditions affecting phosphate exchanges across water-sediment interface.

This study showed a difference in the chemical composition of the two sediments. In the fry pond, the majority of the phosphate was present as inorganic phosphate $(92.5 \%)$, especially in forms bound to calcium. On the other hand, total phosphate was divided equally into organic (49.2\%) and inorganic $(49.8 \%)$ phosphorus in the maturation pond. In this pond, phosphate solubility increased in the interstitial water under anoxic conditions. The phosphate released came principally from the inorganic fraction, particularly from $\mathrm{Fe}(\mathrm{OOH})-\mathrm{P}$. The maturation pond sediments, which had accumulated much organic matter, liberated more orthophosphate than did the fry pond sediments. Under air flushing, phosphate adsorption is significant particularly in the maturation and fry pond sediments.

Keywords: phosphate, maturation pond, fry pond, release, adsorption.

1. Laboratoire d'Écologie Appliquée, Département des Sciences de la vie, Faculté des Sciences et Techniques, BP 523, 23000 Bêni-Mellal, Maroc.

2. Département de Géologie, Faculté des Sciences Agdal, Univ. Med. V, Rabat.

* Correspondance : hasnaoui@fstbm.ac.ma / must hasnaoui@yahoo.com

** Les commentaires seront reçus jusqu'au 31 mai 2006. 


\section{RÉSUMÉ}

Les sédiments utilisés dans cette étude sont prélevés dans deux milieux différents (bassin de maturation du lagunage de Boujaad et étang d'alevinage de la station Deroua, Béni-Mellal) dans le but de comprendre les conditions du milieu qui provoquent les échanges du phosphore à l'interface eau-sédiment et de comparer les échanges du phosphore à ce niveau.

Cette étude a montré une différence dans la composition chimique des deux sédiments. En effet, au niveau de l'étang d'alevinage, le phosphore inorganique représente une part majoritaire $(92,5 \%)$ surtout liée au calcium. En revanche, le phosphore total est réparti en phosphore organique $(49,2 \%)$ et inorganique $(49,8 \%)$ au niveau du bassin de maturation.

Dans le bassin de maturation, le relargage du phosphore est plus important et il est sous forme inorganique lié essentiellement aux hydroxydes de fer. La fixation du phosphore par les sédiments est importante particulièrement en conditions aérobies, aussi bien dans le bassin de maturation que dans l'étang d'alevinage.

Mots clés : phosphore, bassin de maturation, étang d'alevinage, relargage, fixation.

\section{1 - INTRODUCTION}

La mobilisation du phosphore comporte plusieurs processus tels que la désorption, la dissolution du phosphore inclus dans les précipités, les échanges de ligands, le processus de minéralisation achevé par une hydrolyse des liaisons esterphosphates, le relargage du phosphore à partir des cellules vivantes et enfin l'autolyse des cellules (BOSTROM et al., 1988). Elle peut être affectée par plusieurs facteurs tels que la température, l'oxygène dissous, le $\mathrm{pH}$ et la nature des sédiments (BOSTROM et al., 1988 ; GERKE, 1993 ; HOUSE et al., 1995 ; MATEJKA et al., 1992 ; SOLTAN et al., 1993). Ces processus peuvent avoir lieu aussi bien dans la colonne d'eau que dans les sédiments. Les différentes formes du phosphore présentes dans les sédiments peuvent être relarguées directement dans la colonne d'eau (BOSTROM et al., 1988 ; BOERS \& DE BLES, 1991 ; BOERS et al., 1994).

Si les échanges de phosphore inorganique sont aujourd'hui bien compris, les fractions organiques du phosphore le sont moins. L'accumulation de la matière organique, la sédimentation des algues produites dans les écosystèmes aquatiques, suivie de leur dégradation, peut jouer un double rôle, dans la modification des conditions physico-chimiques du sédiment et dans l'apport du phosphore organique dans l'eau surnageante.

Cette étude, réalisée sur des sédiments prélevés dans deux milieux différents (lagunage et étang d'alevinage), a pour objectif de comparer d'une part, les résultats de la spéciation chimique du phosphore et, d'autre part, les échanges du phosphore à l'interface eau-sédiment. 


\section{2 - MATÉRIEL ET MÉTHODES}

\subsection{Sites de l'étude}

Cette étude a été réalisée sur des sites situés dans la région Tadla-Azilal (centre du Maroc). Un bassin de maturation (lagunage de Boujaad) de $5780 \mathrm{~m}^{2}$ de superficie, $1,5 \mathrm{~m}$ de profondeur et un temps de séjour de 7 jours et un étang d'alevinage (station Deroua, Béni-Mellal) d'une surface de $2000 \mathrm{~m}^{2}$ et $1,5 \mathrm{~m}$ de profondeur.

La richesse en éléments nutritifs du bassin de maturation peut être favorable à la production des poissons. Pour cela, le sédiment a été prélevé pour comparer sa composition chimique et sa capacité de relargage et de fixation au sédiment d'un étang d'alevinage.

\section{2 Échantillonnage}

Prélevés au printemps 2000 dans les deux sites à l'aide d'un tube carottier en polychlorure de vinyle (PVC), les sédiments incluent la couche superficielle sur une épaisseur de $5 \mathrm{~cm}$. La spéciation du phosphore a été effectuée selon la méthode de (GOLTERMAN \& DE GROOT, 1994). Les orthophosphates étaient dosés selon la méthode décrite par RODIER (1984).

\subsection{Protocole expérimental des échanges du phosphore à l'interface eau-sédiments sous l'effet de l'oxygène}

\subsubsection{Préparation des sédiments}

Les sédiments prélevés ont d'abord été centrifugés à 3000 tours/min pendant 30 minutes pour éliminer la majeure partie de l'eau interstitielle puis homogénéisés ; ils étaient ensuite soumis aux tests de relargage et de fixation.

\subsubsection{Conditions d'incubation}

Pour chaque sédiment, deux séries de six fioles Erlenmeyer ont été incubées à l'obscurité, dans une chambre thermostatée à $22^{\circ} \mathrm{C}$. La première série est mise en conditions aérobies, l'eau surnageante étant oxygénée en continu grâce à des diffuseurs. La deuxième série est mise en conditions anaérobies, les fioles étant désaérées à l'azote gazeux puis fermées hermétiquement par des bouchons étanches. Trois réplicats ont été réalisés pour chaque essai. L'évolution de la concentration en phosphore dans l'eau surnageante a été suivie en 7 jours par dosage des orthophosphates sur des aliquotes d'eau prélevés après homogénéisation. Les résultats sont exprimés en $\mu \mathrm{g} / \mathrm{g}$ de sédiment sec.

\subsubsection{Tests de relargage et de fixation du phosphore par les sédiments}

Le relargage du phosphore a été étudié au laboratoire, dans deux séries de fioles Erlenmeyer (trois répétitions), avec $5 \mathrm{~g}$ de sédiments dans $250 \mathrm{~mL}$ d'eau distillée, cette concentration ayant été préalablement déterminée pour son aptitude à maximiser le relargage (KASSILA et al., 2001). Pour les tests de fixation, deux séries de fioles Erlenmeyer (trois répétitions), contenant la même concen- 
tration de sédiment par litre d'eau distillée, étaient enrichies par $50 \mathrm{mg}$ P/L préparé à partir de $\mathrm{K}_{2} \mathrm{HPO}_{4}$.

\section{3 - RÉSULTATS ET DISCUSSION}

\subsection{Spéciation du phosphore des sédiments du bassin de maturation et de l'étang d'alevinage}

Les teneurs en phosphore total des sédiments prélevés dans le bassin de maturation du lagunage de Boujaad et de l'étang d'alevinage sont comparables (3243,4 et $2563 \mu \mathrm{g} / \mathrm{g}$ de sédiment sec respectivement) (figure 1). Cependant, la spéciation du phosphore montre une différence entre les deux compartiments minéral et organique.
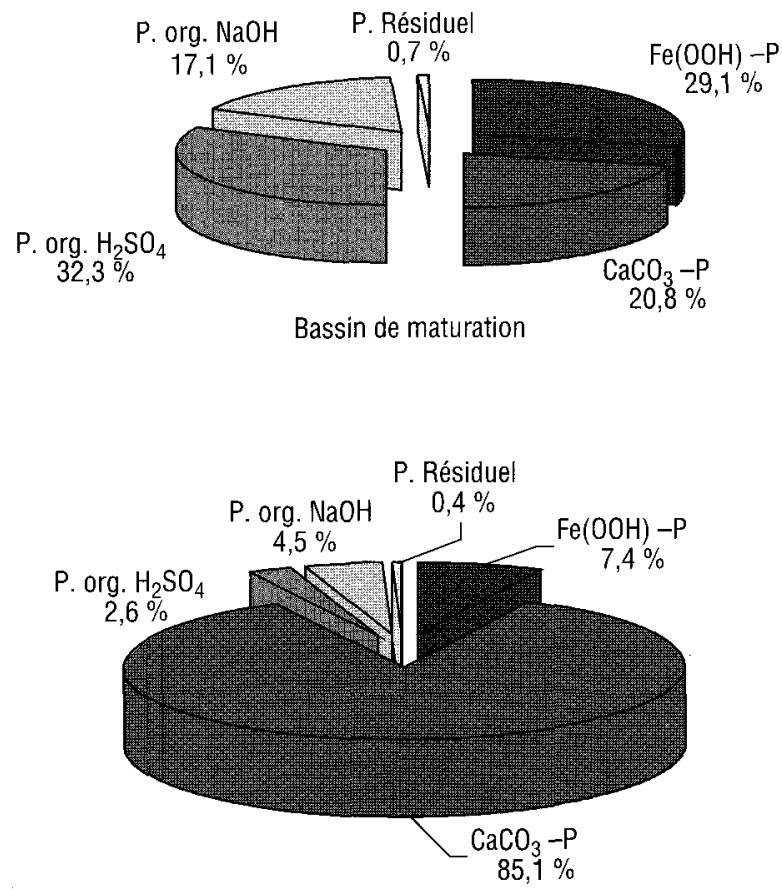

Étang d'alevinage

Figure 1 Résultats de la spéciation du phosphore des sédiments du bassin de maturation (lagunage de Boujaad) et de l'étang d'alevinage (station Deroua) (moyenne de trois replicats).

Phosphorus speciation results in a maturation pond (lagunage of Boujaad) and a fry pond (Deroua fish farm). (Mean values of 3 replicates). 
Dans l'étang d'alevinage, le phosphore minéral représente une part majoritaire $(92,5 \%)$ avec une prédominance du phosphore lié au calcium $(2181 \mu \mathrm{g} / \mathrm{g}$ de sédiment sec) alors que dans le bassin de maturation, le phosphore total est réparti en phosphore minéral $(49,8 \%)$ et phosphore organique $(49,2 \%)$. La fraction minérale est dominée par le phosphore lié au fer avec des teneurs atteignant $945 \mu \mathrm{g} / \mathrm{g}$ de sédiment sec (figure 1).

La différence de répartition du phosphore dans ces deux sites peut s'expliquer par une fixation sélective du phosphore puisque après saturation des sites sur la matière organique, le phosphore entre en liaison avec les hydroxydes de fer et par la suite il s'engage dans des liaisons avec le calcium pour aboutir au complexe $\mathrm{CaCO}_{3}$ (GolterMAN \& DE GROOT, 1994).

La richesse du bassin de maturation en matière organique favorise la fixation du phosphore en part majoritaire sur celle-ci (53\%). Par contre, sa non disponibilité en quantité suffisante dans l'étang (carbone organique ne dépassant pas $14 \mathrm{mg} \mathrm{C} / \mathrm{g}$ sédiment sec ; phosphore organique : $7 \%$ ) fait intervenir le compartiment minéral et surtout le calcium qui se trouve en quantité importante dans cette région calcaire.

\subsection{Comparaison des cinétiques de relargage et de fixation du phosphore par les sédiments}

\subsubsection{Tests de relargage}

En aérobiose, la cinétique de relargage du phosphore par le sédiment du bassin de maturation est décroissante avec des teneurs variant de $64 \mu \mathrm{g} / \mathrm{g}$ de sédiment sec le premier jour d'incubation à $8,4 \mu \mathrm{g} / \mathrm{g}$ de sédiment sec le $7^{\mathrm{e}}$ jour. En revanche, au niveau de l'étang d'alevinage la cinétique de relargage est constante et les teneurs ne dépassent pas $10 \mu \mathrm{g} / \mathrm{g}$ de sédiment sec (figure 2).

En conditions anaérobies, la cinétique de relargage du phosphore par le sédiment prélevé de l'étang d'alevinage est identique à celle observée en aérobiose. Deux pics de phosphore relargué par le sédiment du bassin de maturation sont enregistrés le premier jour $\left(90 \mu \mathrm{g} / \mathrm{g}\right.$ de sédiment sec) et le $7^{\mathrm{e}}$ jour (86 $\mu \mathrm{g} / \mathrm{g}$ de sédiment sec) (figure 2). Le fer ferrique du complexe $\mathrm{Fe}(\mathrm{OOH})$-P est réduit en fer ferreux, libérant ainsi le phosphate. Ce résultat est en accord avec la littérature (BATES \& NEAFUS, 1980 ; BARROIN, 1986 ; BOSTROM et al., 1988 ; MATEJKA et al., 1992 ; OULD EL MEHDI, 1998 ; HASNAOUI et al., 2001). Ce processus est encore plus actif en présence des bactéries. BOERS (1986), COMEAU et al. (1987) et TAM et al. (1992) rapportent que le Fe(OOH)-P est à l'origine du phosphore relargué puisque le fer ferrique est utilisé comme accepteur d'électrons par les bactéries dénitrifiantes.

L'intensité des différents processus biologiques qui se déroulent au niveau de l'écosystème (bassin ou étang), dont la photosynthèse, la respiration et la miméralisation de la matière organique, conditionne le $\mathrm{pH}$ de l'eau. Ceci favoriserait le relargage du phosphore lié au $\mathrm{Fe}(\mathrm{OOH})$ et la précipitation ultérieure de ce phosphore avec les carbonates. De même, l'augmentation de l'activité minéralisatrice favorise l'installation des conditions anoxiques au niveau du sédiment et facilite la désorption du phosphore à partir des hydroxydes de fer. Ainsi, la matière organique agit par l'intermédiaire de variables telles le $\mathrm{pH}$ et le potentiel redox. La décomposition de cette matière organique provoque une 
baisse du $\mathrm{pH}$, responsable de la dissolution de $\mathrm{CaCO}_{3}-\mathrm{P}$ (STUMM \& LECKIE, 1971 ; GOLTERMAN, 1995). Le pH est de 7,2 pour le sédiment de l'étang et il est de 8,3 pour celui du bassin de maturation.
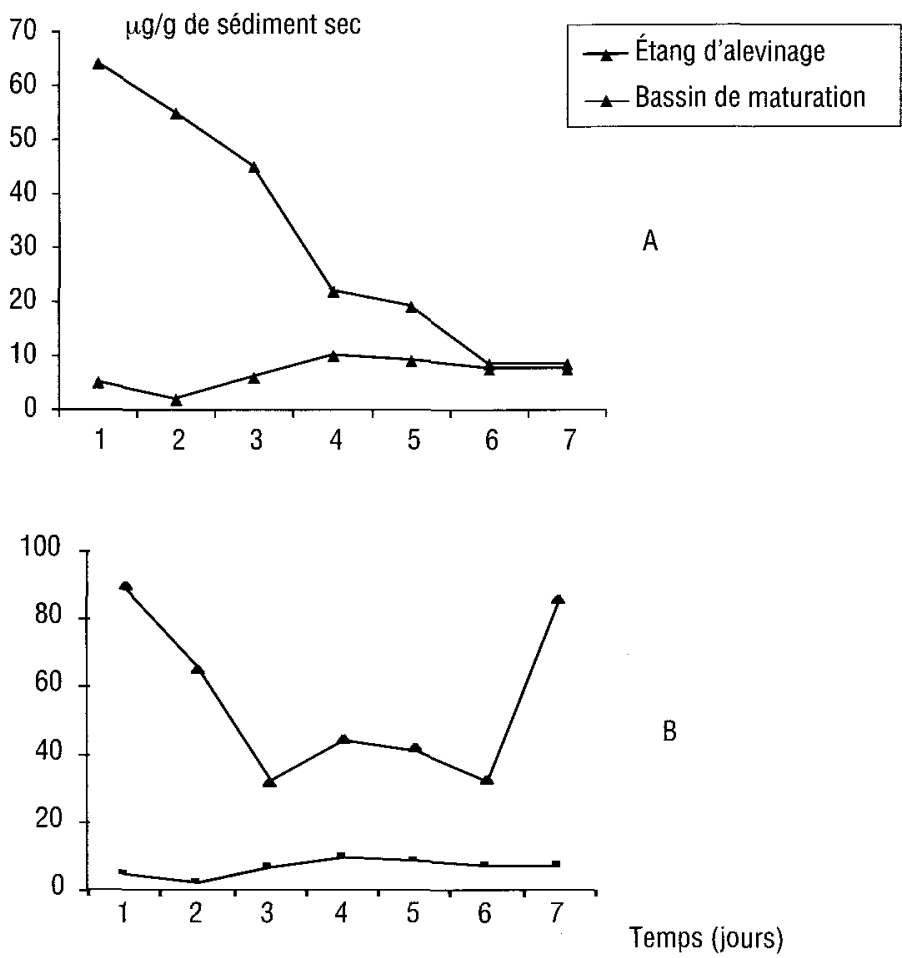

Figure 2 Évolution de la quantité de phosphore relargué par les sédiments du bassin de maturation et de l'étang d'alevinage en conditions aérobies (A) et en conditions anaérobies (B).

Kinetics of phosphate release by maturation and fry pond sediments under oxic $(A)$ and anoxic conditions $(B)$.

\subsubsection{Tests de fixation}

En conditions aérobies, la cinétique de fixation du phosphore par le sédiment de l'étang d'alevinage présente une allure croissante. Les teneurs en phosphore fixées par ce sédiment varient de 350 à $850 \mu \mathrm{g} / \mathrm{g}$ de sédiment sec, alors que celles fixées par le sédiment du bassin de maturation sont plus importantes et presque constantes (de 1258 à $1360 \mu \mathrm{g} / \mathrm{g}$ de sédiment sec). Quoique la quantité du phosphore fixé par le sédiment du bassin de maturation le $1^{\text {er }}$ jour d'incubation soit nettement supérieure, le sédiment de l'étang d'alevinage a fixé une quantité totale plus importante $(500 \mu \mathrm{g} / \mathrm{g}$ de sédiment $\mathrm{sec}$ contre $102 \mu \mathrm{g} / \mathrm{g}$ de sédiment sec fixés par le sédiment du bassin de maturation) (figure 3). 


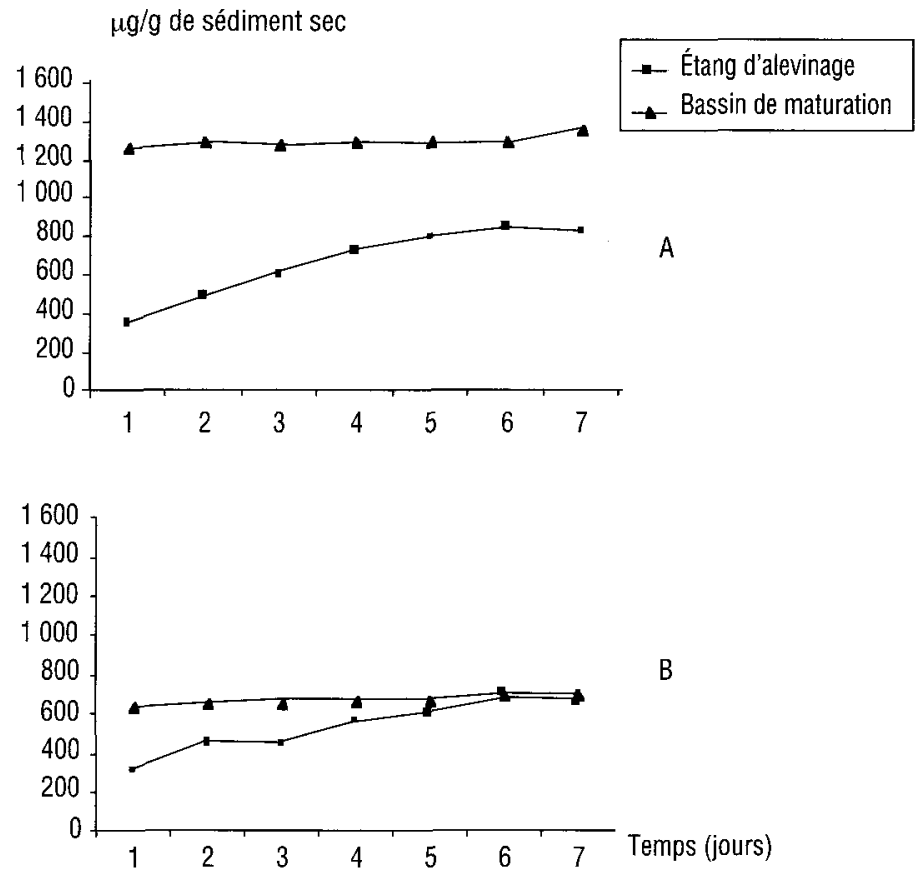

Figure 3 Évolution de la quantité de phosphore fixé par les sédiments du bassin de maturation et de l'étang d'alevinage en conditions aérobies ( $A$ ) et en conditions anaérobies (B).

Phosphate adsorbed onto maturation and fry pond sediments under oxic (A) and anoxic conditions (B).

Tableau 1 Fractionnement chimique des sédiments du bassin de maturation du lagunage de Boujaad et de l'étang d'alevinage de la station Deroua (moyenne de trois replicats).

Table 1 Chemical fractionation of sediment in maturation basin (Boujaad's lagunage) and in fry pond (Deroua fish farm). (Mean values of 3 replicates).

\begin{tabular}{|c|c|c|}
\hline & $\begin{array}{c}\text { Bassin de maturation } \\
\text { ( } \mu \mathrm{g} / \mathrm{g} \text { de sédiment sec) }\end{array}$ & $\begin{array}{c}\text { Étang d'alevinage } \\
\text { ( } \mu \mathrm{g} / \mathrm{g} \text { de sédiment sec) }\end{array}$ \\
\hline P.Total & $3243,4 \pm 85,3$ & $2563 \pm 54,3$ \\
\hline $\mathrm{Fe}(\mathrm{OOH})-\mathrm{P}$ & $945,3 \pm 27,8$ & $188,5 \pm 2,6$ \\
\hline $\mathrm{Ca} \mathrm{CO} 3-\mathrm{P}$ & $674,3 \pm 23,2$ & $2181,2 \pm 48,2$ \\
\hline P. souble dans $\mathrm{H}_{2} \mathrm{SO}_{4}$ & $1046,2 \pm 33,4$ & $65,5 \pm 1,7$ \\
\hline P. souble dans $\mathrm{NaOH}^{4}$ & $553,4 \pm 19,7$ & $117 \pm 7,8$ \\
\hline$P$. résiduel & $24,2 \pm 7$ & $11 \pm 3,1$ \\
\hline
\end{tabular}


Les conditions aérobies ont un effet positif sur l'adsorption du phosphore au niveau du sédiment du bassin de maturation qui est probablement plus riche en FeS que le sédiment de l'étang d'alevinage. L'induction des conditions aérobies entraîne une oxydation de $\mathrm{FeS}$ en $\mathrm{Fe}(\mathrm{OOH})$ capable de fixer du phosphore. Il a été démontré que les bactéries jouent un rôle primordial dans la fixation du phosphore sur $\mathrm{Fe}(\mathrm{OOH})$ en contrôlant l'état d'oxydation du fer (GACHTER et al., 1988 ; DE GROOT \& FABRE, 1993).

Le phosphore non fixé sur $\mathrm{Fe}(\mathrm{OOH})$ est précipité avec les ions calcium de l'eau de manière proportionnelle. GOLTERMAN (1984) a montré que la concentration en ions $\mathrm{Ca}^{2+}$ dans l'eau est un paramètre déterminant dans l'équilibre des orthophosphates entre l'eau et le sédiment. Ce phénomène est observé surtout au niveau du sédiment de l'étang d'alevinage où la concentration de $\mathrm{CaCO}_{3}-\mathrm{P}$ domine.

En anaérobiose, la cinétique de fixation du phosphore par le sédiment du bassin de maturation présente des fluctuations peu importantes (de 645 à 690 $\mu \mathrm{g} / \mathrm{g}$ de sédiment $\mathrm{sec}$ ). Par contre, les teneurs fixées par le sédiment de l'étang oscillent entre 310 et $700 \mu \mathrm{g} / \mathrm{g}$ de sédiment sec.

\section{5 - CONCLUSION}

La spéciation du phosphore des sédiments du bassin de maturation et de l'étang d'alevinage a mis en évidence une différence dans la composition chimique de ces sédiments. En effet, le phosphore du sédiment du bassin de maturation est réparti en fractions organiques et inorganiques, alors que dans l'étang, le phosphore est surtout minéral (92,5\%) lié particulièrement au calcium.

Cette étude a révélé également que les conditions aérobies et anaérobies contrôlent les cinétiques de relargage et de fixation du phosphore dans les sédiments des deux milieux. La comparaison des cinétiques de relargage nous a permis de conclure que contrairement au bassin de maturation, l'oxygénation ne présente aucune influence sur le relargage du phosphore par le sédiment de l'étang. Les quantités du phosphore fixées sont plus importantes en conditions aérobies, aussi bien pour le bassin de maturation que pour l'étang, alors que la quantité totale fixée par le sédiment de l'étang est plus importante.

\section{6 - REMERCIEMENTS}

Les auteurs remercient H.L. Golterman pour son aide scientifique. 


\section{RÉFÉRENCES BIBLIOGRAPHIQUES}

BARROIN G., 1986. Relation phosphoreoxygène : un outil mathématique pour caractériser le métabolisme phosphoré des lacs profonds. Revue Sciences de l'Eau, 5, 29-42.

BATES M.H., NEAFUS N.J.E., 1980. Phosphorus release from sediments from lake Carl Black Well, Oklahoma. Water Research, 14, 1477-1481.

BOERS P., 1986. Studying the phosphorus release from the Loosdrecht lakes sediments using a continuous flow system. Hydrobiological Bull., 20, 51-60.

BOERS P., De BLES F., 1991. Ion concentrations in interstitial water as indicators for phosphorus release: processes and reactions. Water Research, 25, 591-598.

BOERS P., VAN DER BOES J., QUAAK M., VAN DER VLUGT J., 1994. Phosphorus fixation with iron (III) chloride. A new method to combat internal phosphorus loading in shallow lakes. Arch. Hydrobiol., 129, 339-351

BOSTROM B., ANDERSEN J.M., FLEISHER S., JANSSON M., 1988. Exchange of phosphorus across the sediment-water interface. Hydrobiologia, 170, 229-244.

COMEAU Y., RABIONWITZ B., HALL K.J., OLDHAM W.K., 1987. Phosphorus release and uptake in enhanced biological phosphorus removal from wastewater. J. Water Pollut. Contr. Feder., 59, 707-715.

DE GROOT C. J., FABRE A., 1993. The impact of dessication of a freshwater marsh (Garcines Nord, Camarague, France) on sediment-water-vegetation interactions. Part 3 : The fractional composition and the phosphate adsorption characteristics of the sediment. Hydrobiologia, 252, 105-116.

GACHTER R., MEYER J.S., MARES A., 1988. Contribution of bacteria to release and fixation of phosphorus in lake sediments. Limnol. Oceanogr., 33, 15421558.

GERKE J., 1993. Phosphate adsorption by humic/Fe-oxide mixtures aged at $\mathrm{pH} 4$ and $\mathrm{pH} 7$ and by poorly ordered $\mathrm{Fe}$ oxide. Geoderma, 598, 279-288.
GOLTERMAN H.L., 1984. Sediments, modifying and equilibrating factors in the chemistry of freshwater. Verh. Internat. Verein. Limnol., 22, 23-59.

GOLTERMAN H.L., DE GROOT C.J., 1994. Nouvelles connaissances des formes du phosphate : Conséquences sur le cycle du phosphate dans les sédiments des eaux douces peu profondes. Annal. Limnol., 30, 221-232.

GOLTERMAN H.L., 1995. Theoretical aspects of the adsorption of orthophosphate onto iron hydroxide. Hydrobiologia, 315, 5968.

HASNAOUI M., KASSILA J., LOUDIKI M., DROUSSI M., BALVAY G., BARROIN G., 2001. Relargage du phosphore à l'interface eau-sédiment dans des étangs de pisciculture de la station Deroua (Béni Mellal, Maroc). Rev. Sci. de l'Eau, 14(3), 307-322.

HOUSE W.A., DENISON F.H., ARRMITAGE P.D., 1995. Comparison of the uptake of inorganic phosphorus to a suspended and stream bed-sediment. Water Research, 29, 767-779.

KASSILA J., HASNAOUI M., DROUSSI M., LOUDIKI M., YAHYAOUI A., 2001. Relation between phosphate and organic matter in fish-pond sediments of the Deroua fish farm (Beni-Mellal, Morocco): implications for pond management, Hydrobiologia, 450, 57-70.

MATEJKA G., FEUILLADE G., HEULOT I., LEMEHAUTE P., MAZET M., 1992. Les échanges de phosphore à l'interface eau-sédiments- Etudes et mémoires. Tribune de l'eau, 556, 19-25.

OULD EL MEHDI M., 1998. Contribution à l'étude du fonctionnement du lagunage naturel - cas de la station expérimentale de Marrakech - Étude du cycle de l'azote et du phosphore. Thèse de $3^{e}$ cycle, Univ. Cadi Ayyad, Marrakech. 170 p.

RODIER J., 1984. Analyse de l'eau, eaux naturelles, eaux résiduaires, eaux de mer. $7^{e}$ Ed; Dunod, $1365 \mathrm{p}$.

SOLTAN S., ROMER W., ADGO E., GERKE J., SCHILLING G., 1993. Phosphate sorption by Egyptian, Ethiopian \& German soils and $\mathrm{P}$ uptake by rye (Secale 
cereale L.) seedlings. Z. Pflanzenernähr. Bodenk., 156, 501-506.

STUMM W., LECKIE J., 1971. Phosphate exchange with sediments. Proc. $5^{\text {th }}$ Inst. Conf. Water Pollution, DubendorfZurich Pergamon Press, NY. Separatum 406, 1-26.
TAM N.F.Y., WONG Y.S., LEUNG G., 1992. Effect of exogenous carbon sources on removal of inorganic nutrient by the nitrification-denitrification process. Water Research, 26, 1229-1236. 